\title{
Refining Mobile UML State Machines
}

\author{
Alexander Knapp ${ }^{1}$, Stephan Merz ${ }^{2}$, and Martin Wirsing ${ }^{1}$ \\ 1 Institut für Informatik, Ludwig-Maximilians-Universität München \\ $\{$ knapp, wirsing\}einformatik. uni-muenchen.de \\ 2 INRIA Lorraine, LORIA, Nancy \\ Stephan.Merz@loria.fr
}

\begin{abstract}
We study the semantics and refinement of mobile objects, considering an extension of core UML state machines by primitives that designate the location of objects and their moves within a network. Our contribution is twofold: first, we formalize the semantics of state machines in MTLA, an extension of Lamport's Temporal Logic of Actions with spatial modalities. Second, we study refinement concepts for state machines that are semantically justified in MTLA.
\end{abstract}

\section{Introduction}

Software development for mobile computing and mobile computations requires appropriate extensions of the traditional methods and concepts for more traditional system models. Moreover, the correctness and security of implementations of systems based on mobile code presents a major concern, as mobile agents may roam the network and must be guaranteed to work reliably in different locations and in different environments.

In this paper, we attempt to combine semi-formal modeling techniques for mobile systems with formal semantics and refinement. For modeling, we consider an extension of state machines in the "Unified Modeling Language" (UML [14]) for mobility. We first formalize the semantics of mobile state machines in MTLA [11], an extension of Lamport's Temporal Logic of Actions [9] with spatial modalities. Building on this logical semantics, we study refinement concepts for mobile state machines. In particular, we consider two notions of spatial refinement: the first one provides for an object to be split into a hierarchy of cooperating objects. The second one can be used to justify implementations of some high-level object by a set of objects that need not reside at the same location.

There has been much interest in formalizing concepts of UML as well as in semantic foundations for mobile computations, and we mention only the most closely related work. Deiß [6] suggested an encoding of (Harel) Statecharts in TLA, without considering either mobility or refinement. Several formal models of mobile computation have been proposed, either in the form of calculi as in $[5,12]$ or of state machine models as in [8], and sometimes accompanied by logics to describe system behavior [4,13], but we are not aware of refinement notions for mobile computation. Our definitions of refinement of state machines are partly inspired by $[15,16]$; a related notion has been elaborated in [17]. 

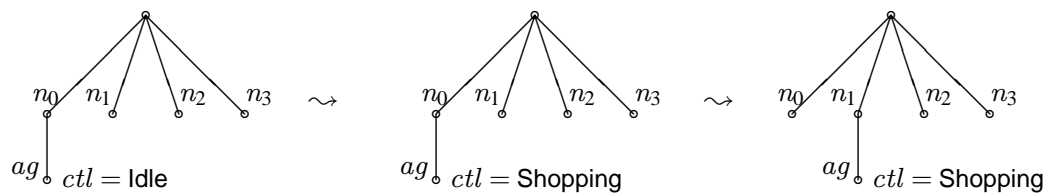

Fig. 1. Prefix of a run.

\subsection{Mobile UML}

Mobile UML [2,3,10] extends UML [14] by concepts for modeling mobile computation. The extension is described in terms of the UML itself, using stereotypes and tagged values as meta-modeling tools. Most importantly, instances of classes distinguished by the stereotype «location» denote locations where other objects may reside. Mobile objects are instances of classes with the stereotype «mobile» and may change their locations over life-time. An actual movement of a mobile object is performed by a move action that takes the target location as its parameter.

\subsection{MTLA}

The logic MTLA [11] is an extension of Lamport's Temporal Logic of Actions [9] intended for the specification of systems that rely on mobility of code. Due to space restrictions, we refer to [11] for precise definitions of its syntax and semantics and only recall the basic intuitions and notations.

Following the intuition of the Ambient calculus [5] due to Cardelli and Gordon, we represent a configuration of a mobile system as a finite tree of nested locations. In this view, mobility is reflected by modifications of the location hierarchy, as agents move in and out of nested domains. Unlike in the Ambient calculus, we assume that locations carry unique ("physical") names. Moreover, instead of endowing each node of a configuration tree with a process, MTLA associates a local state with every node. A run is modeled as an $\omega$-sequence of configuration trees. For example, Fig. 1 shows three configurations of a system run. The transition from the first to the second configuration models a local action that changes the value of the local attribute $c t l$ associated with location $a g$. The second transition represents a move of $a g$ from the location $n_{0}$ to the location $n_{1}$.

The logic MTLA contains both temporal and spatial modalities. Its formulas are evaluated over runs, at a given location. Temporal modalities refer to the truth value of formulas at suffixes of a run. For example $\square F$ asserts that $F$ holds of all suffixes of the run, at the current location.

Similarly, spatial modalities shift the spatial focus of evaluation, referring to locations below the current one. For example, the formula $m[F]$ asserts that $F$ is true of the current run when evaluated at location $m$, provided such a location occurs (strictly and at arbitrary depth) below the current location, otherwise $m[F]$ is trivially satisfied. The dual formula $m\langle F\rangle$ asserts that the location $m$ occurs beneath the current location, and that $F$ holds there. For example, the run of Fig. 1 satisfies the formula $a g[c t l=\mid d l e]$ 
at the root location. We frequently use a more convenient dot notation to refer to local attributes at a given location and write, e.g., ag.ctl = Idle.

As in TLA, we use formulas to describe systems as well as their properties. State transitions are specified using transition formulas that contain primed symbols, as in $a g . c t l=$ Idle $\wedge a g . c t l^{\prime}=$ Shopping. When $P$ is a state formula (i.e., without primed symbols), we write $P^{\prime}$ for the transition formula obtained by replacing all flexible symbols by their primed counterparts; intuitively, this formula asserts that $P$ holds of the successor state. MTLA adds a transition formula $\alpha . n \gg \beta . n$ where $n$ is a name and $\alpha$ and $\beta$ are sequences of names. This formula asserts that the subtree rooted at name $n$ within the tree indicated by $\alpha$ moves below the path $\beta$. The next-state relation of a system is specified by the temporal formula $\square[A]_{v}$ asserting that every transition that modifies the expression $v$ must satisfy the action formula $A$. Similarly, $\square[A]_{\alpha . n}$, where $n$ is a name and $\alpha$ is a sequence of names stipulates that every transition that removes or introduces location $n$ below the subtree indicated by $\alpha$ must satisfy $A$.

Hiding of state components can be expressed in MTLA using existential quantification. For example, $\exists a g . c t l: F$ holds if one can assign some value to the attribute ctl of location $a g$ at every state such that $F$ holds of the resulting run. As in TLA, the precise definition is somewhat more complicated in order to preserve invariance under stuttering. One may also quantify over names and write $\exists n: F$; this hides the name as well as all its attributes. These quantifiers observe standard proof rules. In particular, we have the introduction axioms

$$
\begin{aligned}
& \text { (ヨ-ref) } \quad F\left\{t / n, t_{1} / n . a_{1}, \ldots, t_{k} / n . a_{k}\right\} \Rightarrow \exists n: F \\
& \text { ( } \exists \text {-sub) } \quad m\langle\text { true }\rangle \Rightarrow \exists n: m . n\langle\text { true }\rangle \quad(m \not \equiv n)
\end{aligned}
$$

The axiom ( $\exists$-ref) asserts that $\exists n: F$ can be derived by finding a "spatial refinement mapping" that substitutes witnesses for the hidden name $n$ as well as for its attributes. The axiom $(\exists$-sub) allows us to introduce a new sublocation $n$ of an existing location $m$.

\section{Statecharts and their MTLA Semantics}

We introduce state machines for mobile objects and provide them with a formal semantics based on MTLA. Our concepts are illustrated by means of the "shopper" example: A mobile shopping agent is sent out to gather offers for some item in several shops; when returning to its home base, the shopping agent presents the offers that it has found.

\subsection{State Machines for Mobility}

UML state machines, an object-oriented variant of Statecharts as defined by Harel [7], are an expressive and feature-rich class of state transition systems with a complex semantics [18]. In this paper, we consider a restricted class of state machines, but extended by a special move action. In particular, we consider neither hierarchical nor pseudostates, with the exception of a single initial state per state machine. We consider only events triggered by asynchronous signals (excluding call, time, and change events) and 


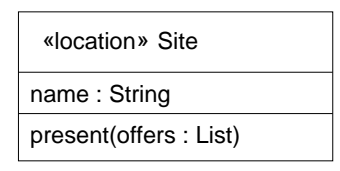

\begin{tabular}{|l|}
\hline «mobile» Shopper \\
\hline name : String \\
home : Site \\
\hline $\begin{array}{l}\text { look(i : Item) } \\
\text { offer(o: Offer) }\end{array}$ \\
\hline
\end{tabular}

(a) Class diagram.

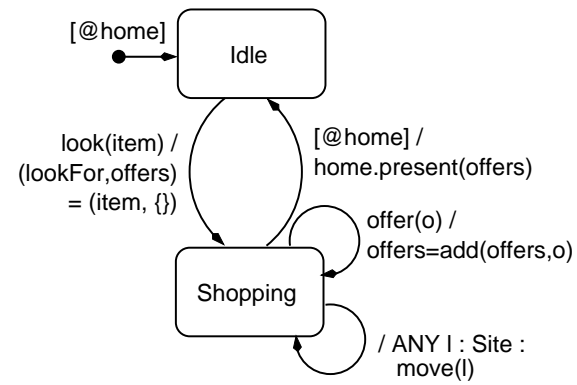

(b) State machine for the shopper.

Fig. 2. High-level model for the shopper.

ignore deferred events. Although our encoding could be extended to encompass all features of UML state machines, the simplifications we impose let us concentrate on the problems of mobility and refinement that are our primary concern.

Transitions of state machines carry labels of the form $\operatorname{trig}[\mathrm{grd}] / \mathrm{act}$, any and all of which can be absent. The trigger trig denotes a signal receptions of the form op $(p a r)$ where op is the name of an operation declared in the class and par is a list of parameters. The guard grd is a Boolean expression over the attributes of the class and the parameters that appear in the trigger clause. In addition, we allow for guards $e_{1} \prec e_{2}$ that refer to the hierarchy of objects; such a clause is true if (the object denoted by) $e_{1}$ is currently located beneath $e_{2}$. The most common form is self $\prec e$, requiring the current object to be located below $e$, which we abbreviate to @e. The action act denotes the response of an object, beyond the state transition. For simplicity, we assume that all actions are of the form ANY $x: P:$ upd; send; move where each of the constituents may be absent. Herein, $P$ is a predicate over location objects, and ANY $x: P$ functions as a binder that chooses some location object $x$ satisfying $P$ which can be used in the remainder of the action. The upd part is a simultaneous assignment $\left(a_{1}, \ldots, a_{k}\right)=\left(e_{1}, \ldots, e_{k}\right)$ of expressions $e_{i}$ to attributes $a_{i}$. The send part is of the form $e .0 p(p a r)$ and denotes the emission of a signal op with parameters par to receiver object $e$. Finally, the move part consists of a single move $(e)$ action that indicates that the object should move to the location object whose identity is denoted by $e$. We require that all free variables in the action are among the attributes of the class, the parameters introduced by the trigger, and the location $x$ bound by ANY. Figure 2(b) shows a first state machine for our shopping agent, based on the class diagram of Fig. 2(a). For the subsequent refinements, we will not explicitly indicate the class diagrams, as they can be inferred from the elements that appear in the state machines.

Our interpretation of transitions deviates in certain ways from the UML standard. First, the UML standard prioritizes triggerless transitions (so-called "completion transitions") over transitions that require an explicit triggering event. In contrast, we consider that completion transitions may be delayed; this less deterministic interpretation is more appropriate for descriptions at higher levels of abstraction. As a second, minor deviation, we allow guards to appear in transitions leaving a state machine's initial state. 


\subsection{MTLA Semantics of State Machines}

We formalize systems of interacting, mobile state machines in MTLA. The formalization enables us to prove properties about systems specified in UML. We will also use it to justify correctness-preserving refinement transformations.

In MTLA, every object is represented by a MTLA location whose local state includes a unique, unmodifiable identifier self. We denote by $O b j$ the set of all MTLA locations that represent objects of a given object system. The subset $L o c$ denotes the set of MTLA locations that represent UML Location objects (including Mobile Locations), and the formalization of a system of state machines at a given level of abstraction is with respect to these sets $O b j$ and $L o c$. An object configuration is represented as a tree of names as described in Sect. 1.2.

The local state at each node represents the attributes of the corresponding object, including self. In addition, we use the attributes $c t l$ to hold the current control state of the object (i.e., the active state of the corresponding state machine) and evts to represent the list of events that are waiting to be processed by the object. Objects interact asynchronously by sending and receiving messages. In the MTLA formalization, the communication network is represented explicitly by an attribute msgs located at the root node of the configuration tree.

Every transition of an object is translated into an MTLA action formula that takes a parameter $o$ denoting the location corresponding to the object. For lack of space, we do not give a precise, inductive definition of the translation, but only indicate its general form. In the following, if $\varphi$ is an MTLA expression (a term or a formula), we write $\varphi^{x}$ and $\varphi_{o}$, respectively, for the expressions obtained by replacing $x$ by $x$. self and by replacing all attributes $a$ of $o$ by $o . a$.

The action formula representing a transition is a conjunction built from the translations of its trigger, guard, and action components. The automaton transition from states src to dest is reflected by a conjunct $o . c t l=\operatorname{src} \wedge o . c t l^{\prime}=$ dest.

A trigger $\mathrm{op}($ par $)$ contributes to the definition of the action formula in two ways: first, the parameters par are added to the formal parameters of the action definition. Second, we add the conjunct

$$
\neg \text { empty }(\text { o.evts }) \wedge \text { head }(\text { o.evts })=\langle\mathrm{op}, \text { par }\rangle \wedge \text { o.evts }{ }^{\prime}=\operatorname{tail}(\text { o.evts })
$$

asserting that the transition can only be taken if the trigger is actually present in the event queue and that it is removed from the queue upon execution of the transition. For transitions without an explicit trigger we add the conjunct UNCHANGED o.evts to indicate that the event queue is unmodified.

A Boolean guard $g$ over the object's attributes is represented by a formula $g_{o}$, indicating that $g$ is true at location $o$. A constraint $e_{1} \prec e_{2}$ on the hierarchy of objects is represented by a conjunct of the form

$$
\bigvee_{o_{1}, o_{2} \in O b j} o_{1} . \text { self }=\left(e_{1}\right)_{o} \wedge o_{2} . \text { self }=\left(e_{2}\right)_{o} \wedge o_{2} . o_{1}\langle\text { true }\rangle
$$

The representation of an action consists of action formulae for multiple assignment, message sending, and moving. If an action shows an ANY $x: P$ quantifier the conjunction acts of these formulae are bound by a disjunction $\bigvee_{x \in L o c} P_{o}^{x} \wedge a c t s^{x}$. In more 
detail, a multiple assignment to attributes is represented by a formula

$$
o . a_{1}^{\prime}=\left(e_{1}\right)_{o}^{x} \wedge \ldots \wedge \text { o. } a_{k}^{\prime}=\left(e_{k}\right)_{o}^{x} \wedge \mathrm{UNCHANGED}\left\langle o . a_{k+1}, \ldots, o . a_{n}\right\rangle
$$

where $a_{k+1}, \ldots, a_{n}$ are the attributes of $o$ that are not modified by the assignment and where $x$ is the variable bound by ANY. Sending a message $e .0 \mathrm{p}(p a r)$ is modeled by adding a tuple of the form $\left\langle e_{o}^{x}\right.$, op, par $\left._{o}^{x}\right\rangle$ to the network msgs. For actions that do not send a message we add the conjunct $m s g s^{\prime}=m s g s$. If the action contains a clause move $(e)$, we add a conjunct

$$
\bigvee_{l \in \text { Loc }} \text { l.self }=e_{o}^{x} \wedge \varepsilon . o \gg l . o
$$

that asserts that $o$ will move to (the location with identity) $e_{o}$. Otherwise we add the conjunct $\bigwedge_{l \in L o c}[\text { false }]_{l . o}$, which abbreviates $\bigwedge_{l \in L o c}(l . o\langle$ true $\rangle \Leftrightarrow O l . o\langle$ true $\rangle)$, to indicate that the object does not enter or leave any location in $L o c$.

To model the reception of new events by the object, we add an action $\operatorname{RcvEvt}(o, e)$ that removes an event $e$ addressed to $o$ from the network and appends it to the queue evts of unprocessed events while leaving all other attributes unchanged. We also add an action DiscEvt $(o)$ that discards events that do not have associated transitions from the current control state. The entire next-state relation $N \operatorname{ext}(o)$ of object $o$ is represented as a disjunction of all actions defined from the transitions and the implicit actions RcvEvt and DiscEvt, existentially quantifying over all parameters that have been introduced in the translation.

A state predicate Init $(o)$ defining the initial conditions of object $o$ is similarly obtained from the transition from the initial state of the state machine. Finally, the overall specification of the behavior of an object $o$ of class $C$ is given by the MTLA formulas

$$
\begin{aligned}
I C(o) \equiv & \wedge \operatorname{Init}(o) \wedge \text { o.evts }=\langle\rangle \wedge \square[N \operatorname{ext}(o)]_{\text {attr }(o)} \wedge \square[\text { false }]_{\text {o.self }} \\
& \wedge \wedge_{l \in L o c} \square[N \operatorname{ext}(o)]_{l . o} \\
C(o) \equiv & \exists \text { o.ctl,o.evts }: I C(o)
\end{aligned}
$$

The "internal" specification $I C(o)$ asserts that the initial state must satisfy the initial condition, that all modifications of attributes of $o$ and all moves of $o$ (entering or leaving any location of $L o c$ ) are accounted for by the next-state relation, and that the object identity is immutable. Here, $\operatorname{attr}(o)$ denotes the tuple consisting of the explicitly declared attributes and the implicit attributes $c t l$ and $e v t s$. For example, the formula IShopper $(a g)$ shown in Fig. 3 defines the behavior of an object $a g$ of class Shopper introduced in Fig. 2(b). The "external" specification $C(o)$ is obtained from $I C(o)$ by hiding the implicit attributes $c t l$ and evts.

The specification of a finite system of objects consists of the conjunction of the specifications of the individual objects. Moreover, we add conjuncts that describe the hierarchy of locations and objects and that constrain the network. For our shopper example, we might assume a typical system configuration being given by the object diagram 


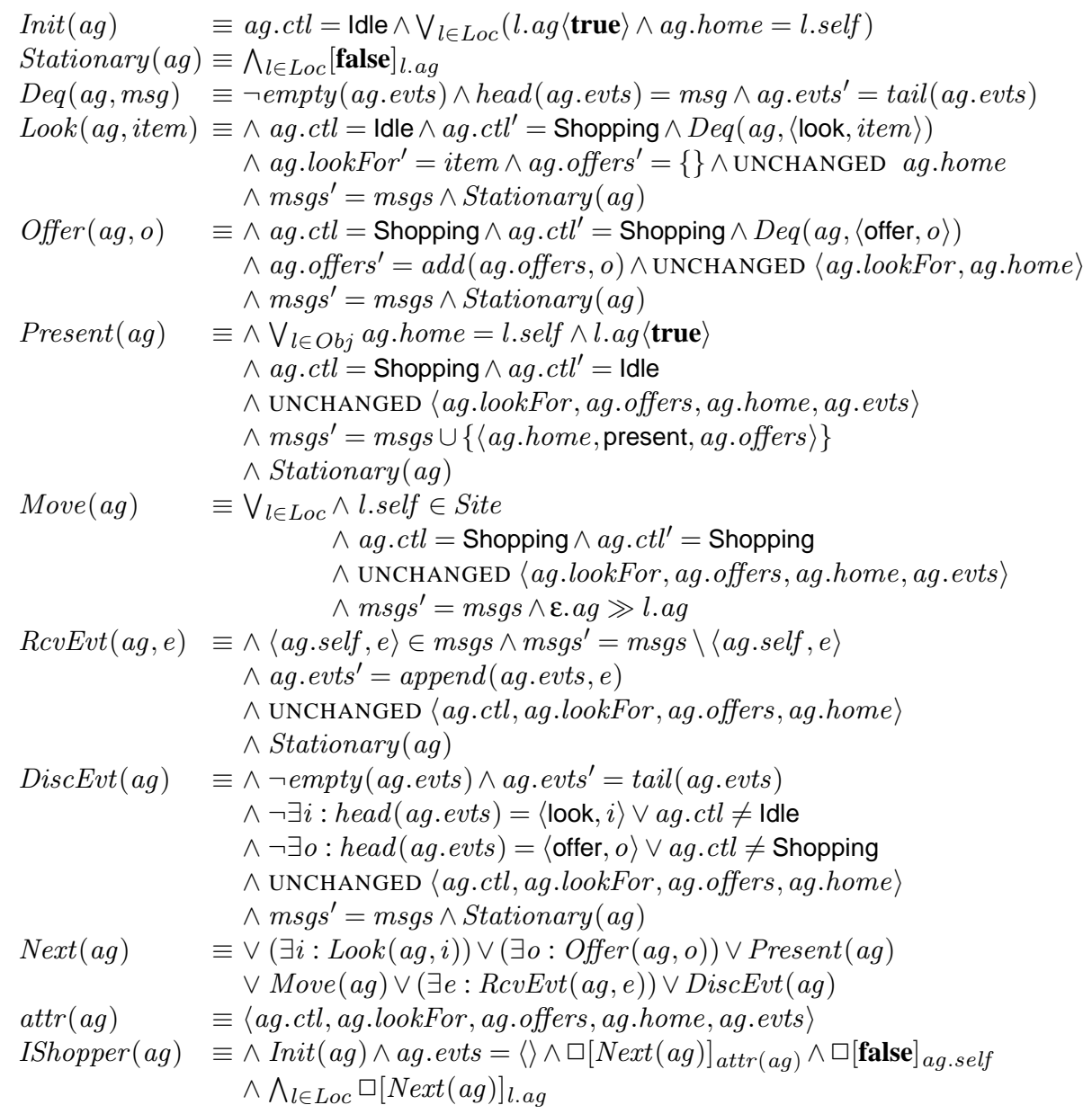

Fig. 3. MTLA specification of the shopper behavior (see Fig. 2(b))

in Fig. 4. This configuration can be translated into the formula

$$
\begin{aligned}
& \text { Sys } \equiv \exists m s g s: \wedge \bigwedge_{i=1}^{N} s_{i}\left\langle\text { self }=\text { shop }-i \wedge j o e[\text { false }] \wedge \bigwedge_{j=1}^{N} s h_{j}[\text { false }]\right\rangle \wedge \operatorname{Site}\left(\text { sh }_{i}\right) \\
& \wedge \text { joe }\left\langle\text { self }=\text { joe } \wedge \bigwedge_{i=1}^{N} \operatorname{sh} h_{i}[\text { false }]\right\rangle \wedge \operatorname{Site}(\text { joe }) \\
& \wedge \text { joe.ag }\langle\text { self }=\text { shopper }\rangle \wedge \text { Shopper }(a g) \\
& \wedge \bigwedge_{l \in L o c} \square[\text { false }]_{l . s h_{1}, \ldots, l . s h_{N}, l . j o e} \\
& \wedge \operatorname{msgs}=\langle\rangle \wedge \square\left[\bigvee_{o \in O b j} \operatorname{Next}(o)\right]_{m s g s}
\end{aligned}
$$

The formula in the scope of the existential quantifier asserts that the configuration contains the $N+1$ sites $s h_{1}, \ldots, s h_{N}$ and joe, and a shopping agent $a g$. Moreover, joe and the shops are immobile and unnested locations, whereas $a g$ is situated beneath joe. The last conjunct asserts that messages are only sent and received according to the specifications of the participating objects. The external specification is obtained by 


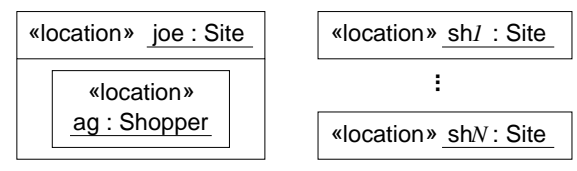

Fig. 4. Object diagram for the shopper example.

hiding, via existential quantification, the set of messages in transit, which is implicit at the UML level.

For this example, $O b j$ is the set $\left\{s h_{1}, \ldots, s h_{N}, j o e, a g\right\}$ and $L o c=O b j \backslash\{a g\}$. Moreover, we define a set Site containing the identities of the elements of Loc, i.e. Site $=\{$ shop- $1, \ldots$, shop $-N$, joe $\}$.

One purpose of our formalization is to prove properties about a system of objects. For the shopper example, we can deduce that the shopping agent is always located at its home agent or at one of the shops, expressed by the formula

$$
\square\left(\bigvee_{l \in L o c} l . a g\langle\text { true }\rangle\right)
$$

\section{Refinement of state machines}

In an approach based on refinement, interesting correctness properties of systems can already be established for models expressed at a high level of abstraction. Subsequent models introduce more detail, but ensure that all properties are preserved. In this paper, we focus on the refinement of state machines, and we add a "spatial" dimension to refinement that allows a designer to introduce more structure in the object hierarchy. In particular, a single high-level object can be refined into a tree of sub-objects. Throughout, we assume that the public interface of a refining class contains that of the refined one, and that the sets $O b j$ and $L o c$ of objects and Location objects of the refining model are supersets of those of the refined model.

\subsection{Interface Preserving Refinement}

Usually, early system models afford a high degree of non-determinism, which is reduced during system design. For example, consider the state machine for the shopping agent shown in Fig. 5, which imposes a number of constraints with respect to the state machine shown in Fig. 2(b). After arriving at a new shop location (whose identity is recorded in the additional attribute loc), the agent may now either query for offers by sending a new message getOffer or it may immediately move on to another neighbor location. In the former case, the agent waits until the offers are received, adds them to its local memory, and then moves on. When the agent arrives at its home location, it may quit the cycle, presenting the collected offers and returning to the Idle state.

Intuitively, the state machine of Fig. 5 is a refinement of the one shown in Fig. 2(b) because the states of the refined state machine can be mapped to those of the high-level state machine such that every transition of the lower-level machine either is explicitly 


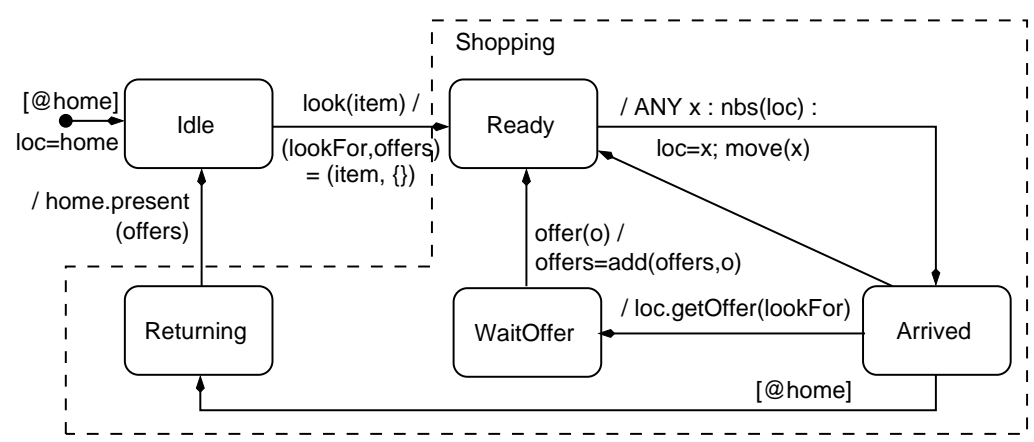

Fig. 5. Refined state machine for the shopper.

allowed or is invisible at the higher level. In particular, the states Ready, Arrived, WaitOffer, and Returning can all be mapped to the high-level state Shopping, as indicated by the dashed line enclosing these states. Assuming that the set $\operatorname{nbs}(s)$ contains only identities in Site, for all $s \in$ Site, each transition of the refined model either corresponds to a transition of the abstract model or to a stuttering transition. For example, the transition from Arrived to WaitOffer is invisible at the level of abstraction of the model shown in Fig. 2(b).

We now formalize this intuition by defining the notion of a state machine $R$ refining another state machine $M$ for a class $C$. Semantically, refinement is represented in linear-time formalisms by trace inclusion or, logically, by validity of implication. However, we will be a little more precise about the context in which $M$ and $R$ are supposed to be embedded. Both machines are specified with respect to attribute and method signatures $\Sigma^{R}$ and $\Sigma^{M}$ that include all method names that appear in transition labels (either received or sent), and we assume that $\Sigma^{R}$ extends $\Sigma^{M}$. Similarly, we assume that the sets $O b j^{R}$ and $L o c^{R}$ of MTLA names for the objects and the Location objects at the level of the refinement are supersets of the corresponding sets $O b j^{M}$ and $\operatorname{Loc}^{M}$ at the abstract level. Finally, the refinement may be subject to global hypotheses about the refined system, such as the hierarchy of names, that are formally asserted by an MTLA state predicate $H$. Thus, we say that the class $R$ with associated state machine formalized by the MTLA formula $C^{R}$ refines class $M$ whose state machine is described by $C^{M}$ under hypothesis $H$ if for all system specifications Sys $^{M}$ and Sys $^{R}$ where Sys $^{R}$ results from $S y s^{M}$ by replacing all occurrences of $C^{M}(o)$ by $C^{R}(o)$ and by conjoining some formulas such that $S y s^{R}$ implies $\square H$, the implication $S y s^{R} \Rightarrow S y s^{M}$ is valid.

In order to prove that $R$ refines $M$, we relate the machines by a mapping $\eta$ that associates with every state $s$ of $R$ a pair $\eta(s)=(\operatorname{Inv}(s), A b s(s))$ where $\operatorname{Inv}(s)$ is a set of MTLA state predicates, possibly containing spatial operators, and where $A b s(s)$ is a state of $M$. With such a mapping we associate certain proof obligations: the invariants must be inductive for $R$, and the (MTLA formalizations of the) transitions of the machine $R$ must imply some transition allowed at the corresponding state of $M$, or leave unchanged the state of $M$. 
Theorem 1. Assume that $M$ and $R$ are two state machines for classes $C^{M}$ and $C^{R}$ such that the attribute and method signature $\Sigma^{R}$ of $C^{R}$ extends the signature $\Sigma^{M}$ of $C^{M}$, and that $\eta$ is a mapping associating with every state $s$ of $R$ a set Inv $(s)$ of MTLA state predicates and a state Abs $(s)$ of $M$. If all of the following conditions hold then $R$ refines $M$ under hypothesis $H$. We write $\bar{\varphi}$ for

$$
\varphi\left\{A b s(\text { o.ctl }) / \text { o.ctl, o.evts } 1_{\Sigma^{M}} / \text { o.evts, } m s g s 1_{\Sigma^{M}} / m s g s\right\}
$$

where $\left.\right|_{\Sigma}$ denotes the subsequence of elements e whose first component is in $\Sigma$.

1. Abs $\left(s_{0}^{R}\right)=s_{0}^{M}$ where $s_{0}^{M}$ and $s_{0}^{R}$ denote the initial states of $M$ and $R$. Moreover,

$$
\models H \wedge \operatorname{Init}^{R}(o) \Rightarrow o\left[\operatorname{Inv}\left(s_{0}^{R}\right)\right] \wedge \overline{\operatorname{Init}^{M}}(o)
$$

holds for the initial conditions Init $^{R}$ and Init $^{M}$ of $M$ and $R$.

2. For every transition of $R$ with source and target states $s$ and $t$ formalized by the MTLA action formula $A(o, p a r)$ :

$$
\models H \wedge H^{\prime} \wedge o[\operatorname{Inv}(s)] \wedge A(o, p a r) \Rightarrow o\left[\operatorname{Inv}(t)^{\prime}\right]
$$

3. For every state $s$ of $R$ and every outgoing transition of $s$ formalized by formula $A(o, p a r)$, let $A b s(s)$ denote the corresponding state of $M$, let $B_{1}\left(o, p_{1} r_{1}\right), \ldots$, $B_{m}\left(o, p_{m}\right)$ be the MTLA formulas for the outgoing transitions of Abs $(s)$, let $\operatorname{attr}^{M}(o)$ be the tuple of attributes defined for $M$ and $L o c^{M}$ the set of locations for M. Then:

$$
\begin{aligned}
\models H \wedge & H^{\prime} \wedge o[\operatorname{Inv}(s)] \wedge A(o, p a r) \Rightarrow \\
& \vee \bigvee_{i=1}^{m}\left(\exists \operatorname{par}_{i}: \overline{B_{i}}\left(o, \operatorname{par}_{i}\right)\right) \\
& \vee \text { UNCHANGED }\left\langle\overline{\operatorname{attr}^{M}}(o), \operatorname{msgs}_{\Sigma^{M}}\right\rangle \wedge \wedge_{l \in L o c} M[\text { false }]_{l . o}
\end{aligned}
$$

Theorem 1 ensures that $R$ can replace $M$, subject to hypotheses $H$. In particular, all properties expressed by MTLA formulas that have been established for the high-level system will be preserved by the implementation.

In order to prove that the state machine of Fig. 5 refines that of Fig. 2(b) (with respect to $H \equiv \forall s \in$ Site $: \operatorname{nbs}(s) \in$ Site) we must define the mapping $\eta$. We have already indicated the definition of the state abstraction mapping Abs. For the mapping Inv, we associate (the MTLA encoding of) @home with state Returning and ag.loc $\in$ Site with all other states. It is then easy to verify the conditions of Theorem 1. In particular, the transitions leaving state Arrived do not modify the shopping agent's attributes, and they do not send messages contained in the original signature. They are therefore allowed by condition (3) of Theorem 1.

Theorem 1 can also be used to justify refinements that modify the spatial hierarchy of locations. Consider the state machine shown in Fig. 6. It is based on the idea that prior to interacting with an object, incoming agents are first placed in a special sublocation for security checking. Instead of a simple, atomic move from one shop to another as in Figs. 2(b) and 5, this version moves the shopping agent first to the "incoming" sublocation of the target location. If the agent is accepted by the host, as modeled by 


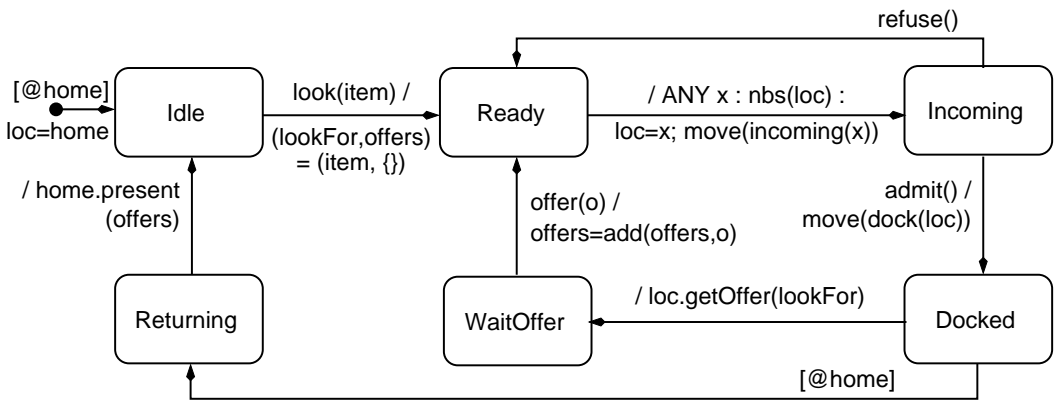

Fig. 6. Spatial refinement of the network sites.

the reception of an admit signal, it transfers to the "dock" sublocation where the real processing takes place. Otherwise, the host will send a refuse signal, and the shopping agent moves on to another neighbor host. Here we assume that every location $l \in L o c$ contains sublocations $l_{-} i n$ and $l_{-} d o c k$. Moreover, we assume functions incoming and dock that look up the id's of the corresponding sub-locations for a given network site.

Formally, Theorem 1 can again be used to show that the "docked" shopper of Fig. 6 is a refinement of that shown in Fig. 5 with respect to the hypothesis

$$
\begin{aligned}
& H \equiv \bigwedge \wedge l . l_{-} \text {in }\langle\text { true }\rangle \wedge l . l_{-} \operatorname{dock}\langle\text { true }\rangle \\
& l \in \text { Loc }^{M} \wedge \operatorname{incoming}(\text { l.self })=l_{\_} \text {in .self } \wedge \operatorname{dock}(\text { l.self })=l_{\_} \text {dock.self }
\end{aligned}
$$

The states Incoming and Docked are mapped to the single high-level state Arrived, and the invariant mapping associates (the MTLA encoding of) @loc with the location Incoming and ag.loc $\in$ Site with all states. Indeed, the move action labeling the transition from the Ready to the Incoming state will be formalized by an MTLA action formula $\bigvee_{l \in L o c^{R}} \varepsilon . a g \gg l_{-} i n . a g$, which implies the corresponding formula $\bigvee_{l \in L o c}{ }^{M} \varepsilon . a g \gg$ l.ag formalizing the move between the high-level states Ready and Arrived, using the hypothesis $H$. Similarly, $H$ and the invariant establish that the move between the Incoming and Docked states maps to a stuttering action: Clearly, the local attributes and the message queue are left unchanged. Moreover, the invariant associated with state Incoming asserts that the agent is located beneath the site (with identity) loc. Therefore, a move to the "dock" sublocation of that same site is invisible with respect to the locations in $L o c^{M}$ : the action implies [false $]_{l . a g}$, for all $l \in L o c^{M}$.

For these kinds of refinement to be admissible, it is essential that the spatial operators of MTLA refer to locations at an arbitrary depth instead of just the children of a node and that it is therefore impossible to specify the precise location of the agent. In fact, we consider the concept of "immediate sublocation" to be as dependent on the current level of abstraction as the notion of "immediate successor state", and MTLA allows to express neither.

\subsection{Interface Refinement I: Spatial Distribution of State}

Frequently, refinements of the spatial hierarchy will be accompanied by a distribution of the high-level attributes over the hierarchy of sublocations of the refined model. For 


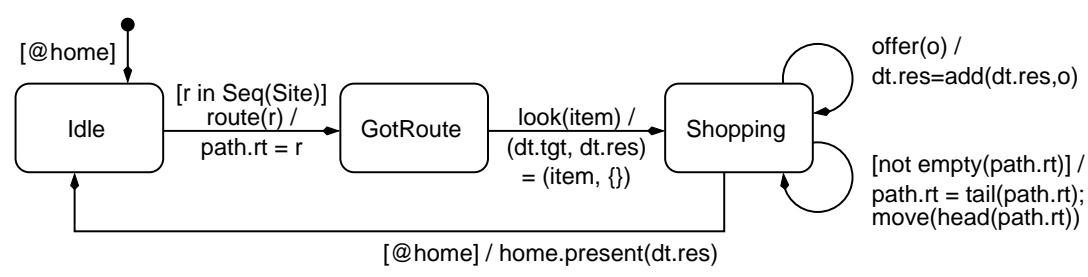

Fig. 7. Spatial refinement of the shopper.

a simple example, departing again from the high-level shopper of Fig. 2(b), consider the state machine shown in Fig. 7. Here we assume that the shopping agent contains two sub-agents path that determines the path to follow through the network and dt that collects the data, and we have replaced the attributes lookFor and offers of the highlevel shopper by attributes tgt and res assigned to the dt sub-agent. ${ }^{3}$ The transition from Idle to GotRoute determines the route of the agent. It is guarded by the condition $r \in \operatorname{Seq}($ Site), asserting that $r$ is a list of (identities of) network sites.

Spatial distribution of attributes is similar to the concept of data refinement in standard refinement-based formalisms. Intuitively, the refinement of Fig. 7 is admissible provided that the public interface is preserved. We will therefore assume that the attributes item and offers have been marked as private in the class diagram for the abstract shopper, ensuring that no other object relies on their presence.

Formally, we modify slightly the MTLA formalization of state machines, taking into account the visibility (either "private" or "public") of attributes. We redefine the external specification of the behavior of an object $o$ of class $C$ with private attributes $a_{1}, \ldots, a_{k}$ as the MTLA formula

$$
C(o) \equiv \exists o . a_{1}, \ldots, o . a_{k}, \text { o.ctl, o.evts: } I C(o)
$$

where $I C(o)$ is defined as before by formula (1). Since the specification of an object system is based on the external object specification, private attributes are invisible at the system level, and the definition of refinement modulo a hypothesis remains as before.

The verification of refinement relies on conditions generalizing those of Theorem 1, provided that the private attributes of the high-level object can be computed from those of the implementation via a refinement mapping [1]. The relation between the two diagrams $R$ and $M$ is therefore given by the mapping $\eta$ as before, complemented by terms $t_{1}, \ldots, t_{k}$ that represent the values of the private high-level attributes $a_{1}, \ldots, a_{k}$. These terms have then to be substituted for the attributes in the formulas concerning the high-level state machine $M$.

Theorem 2. Extending the context of Theorem 1 by terms $t_{1}, \ldots, t_{k}$, we now write $\bar{\varphi}$ for

$$
\varphi\left\{A b s(\text { o.ctl }) / \text { o.ctl, o.evts } 1_{\Sigma^{M}} / \text { o.evts, msgs } 1_{\Sigma^{M}} / m s g s, t_{1} / o . a_{1}, \ldots, t_{k} / o . a_{k}\right\}
$$

\footnotetext{
${ }^{3}$ The renaming of the attributes is not necessary, but will make it clear in the following to which model we are referring.
} 


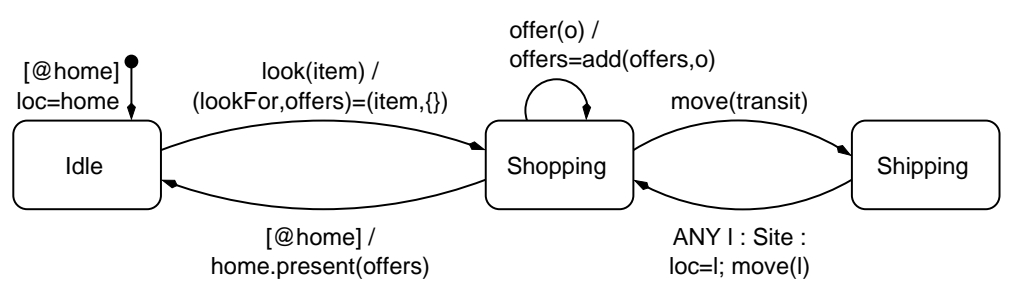

Fig. 8. State machine for the "slow shopper".

If the set of public attributes of $R$ is a superset of those of $M$ then $R$ refines $M$ under hypothesis $H$ up to hiding of attributes o. $a_{1}, \ldots o . a_{k}$ if the conditions of Theorem 1 hold for this new interpretation of substitution.

For the example shown in Fig. 7, the hypothesis is

$$
H \equiv a g . p a t h\langle\text { true }\rangle \wedge a g . d t\langle\text { true }\rangle
$$

The implementation states Idle and GotRoute are both mapped to the abstract state Idle. The invariant mapping assigns the state formula ag.path.rt $\in \operatorname{Seq}($ Site $)$ to the states GotRoute and Shopping. Finally, the refinement mapping is defined by substituting ag.dt.res and ag.dt.tgt for ag.offers and ag.lookFor, respectively. All proof obligations of Theorem 2 are then easily verified.

\subsection{Interface Refinement II: Virtualisation of Locations}

Whereas the notions of spatial refinement that we have considered so far have introduced new (sub-)objects, we have taken care to preserve the hierarchy of the objects present at the abstract levels. Together with the choice of modalities of MTLA, which cannot express the precise location of an object, we have thus been able to represent refinement as implication and to preserve all MTLA properties. However, it can occasionally be desirable to allow for refinements that do not at all times preserve the spatial relationships imposed by the original specification.

For example, the previous specifications of the shopping agent have all assumed that moves between locations happen atomically. Figure 8 presents a variation of the original state machine of Fig. 2(b) where the agent moves to an intermediate transit location, which is not included in Site, before moving to the next site. (A subsequent refinement could add more structure to the transit location, modeling the transport of the agent across the network.) We cannot use Theorems 1 or 2 to prove that this model refines the original one because the move to the transit location cannot be mapped to any high-level action. In fact, the MTLA formula representing the "slow shopper" does not imply the formula encoding the original specification, and the invariant formula (3) asserting that the shopping agent is always located at some location that represents a network site does not hold of the slow shopper.

Such relationships can be formalized by considering a weaker notion of refinement, abstracting from some of the names that occur in the original specification. In our running example, the name of the shopping agent should not actually be part of the interface: the purpose of the system is that the agent's home site learns about offers made by 
other network sites; the use of a mobile agent is an implementation detail. We say that an object system formalized by an MTLA formula $\mathrm{Impl}$ refines another system formalized by $S p e c$ up to hiding of name $n$ if the implication $\operatorname{Impl} \Rightarrow \exists n: S p e c$ holds. In general, the behavior required of object $n$ at the abstract level may be implemented by several implementation objects, hence it does not appear useful to give a "local" rule, similar to Theorems 1 and 2, that attempts to prove refinement by considering a single state machine at a time. Instead, the strategy in proving such a refinement is to define a "spatial refinement mapping", using the rules given in Sect. 1.2. For the slow shopper, we first use rule $\left(\exists\right.$-sub) to introduce a new sublocation, say $l_{-}$virtual, for every high-level location $l$ and then define a refinement mapping that returns the implementation-level agent as long as it is not at the transit location, and otherwise the location $l_{-}$virtual associated with the previous site visited as stored in the attribute loc. The local attributes of the high-level shopper are simply obtained from those of the implementation-level agent. Observe in particular that the invariant (3) cannot be proven of the specification $\exists a g:$ Sys because $a g$ is no longer free in that formula.

Refinement up to hiding of names allows for implementations that differ more radically in structure. For example, the single shopping agent of the initial specification could be implemented by a number of shopping agents that roam the network in parallel, cooperating to establish the shopping list. On the other hand, a correct implementation could also be based on a client-server solution instead of using mobile agents.

\section{Conclusion}

We have studied the applicability of the logic MTLA proposed in [11] in view of formalizing Mobile UML State Machines [3] and of establishing refinement relationships between models described in this language. A configuration of a mobile system is represented as a tree of names, and mobility is reflected by changes to the name hierarchy. MTLA accomodates local attributes at every node in the tree, simplifying the formalization of state-based notations such as UML state machines. The operators of MTLA have been designed to support system refinement; in particular, all spatial operators refer to nodes arbitrarily deep beneath the current node and not just its children as in other spatial logics, e.g. [4].

We have assumed some simplifications and restrictions for our formalization of Mobile UML state machines. In particular, we assume that spatial relationships are specified using constraints $e_{1} \prec e_{2}$, comparing the relative positions of two objects at the current level of abstraction. This assumption has been essential to obtain a sound and elegant representation of refinement as implication of specifications for mobile systems.

Our main objective has been the study of three fundamental refinement principles, focusing on refinements of the spatial hierarchy. We have indicated sufficient conditions for verifying refinement. However, these conditions are incomplete: in particular, it is well known that refinement mappings need to be complemented by devices such as history and prophecy variables in order to obtain completeness [1]. We have also ignored liveness and fairness properties in this paper, and we have mostly restricted ourselves to proving refinement "object by object". We intend to study adequate composition and decomposition concepts in future work. 


\section{References}

1. M. Abadi and L. Lamport. The existence of refinement mappings. Theor. Comp. Sci., 81(2):253-284, May 1991.

2. H. Baumeister, N. Koch, P. Kosiuczenko, P. Stevens, and M. Wirsing. UML for global computing. In C. Priami, editor, Global Computing. Programming Environments, Languages, Security, and Analysis of Systems, volume 2874 of Lect. Notes in Comp. Sci., pages 1-24, Rovereto, Italy, 2003. Springer-Verlag.

3. H. Baumeister, N. Koch, P. Kosiuczenko, and M. Wirsing. Extending activity diagrams to model mobile systems. In M. Aksit, M. Mezini, and R. Unland, editors, Objects, Components, Architectures, Services, and Applications for a Networked World, volume 2591 of Lect. Notes in Comp. Sci., pages 278-293, Erfurt, Germany, 2003. Springer-Verlag.

4. L. Caires and L. Cardelli. A spatial logic for concurrency (part I). Inf. and Comp., 186(2):194-235, Nov. 2003

5. L. Cardelli and A. Gordon. Mobile ambients. Theor. Comp. Sci., 240:177-213, 2000.

6. T. Deiß. An approach to the combination of formal description techniques: Statecharts and TLA. In K. Araki, A. Galloway, and K. Taguchi, editors, Integrated Formal Methods (IFM 1999), pages 231-250, York, UK, 1999. Springer-Verlag.

7. D. Harel. Statecharts: A Visual Formalism for Complex Systems. Science of Computer Programming, 8(3):231-274, 1987.

8. T. A. Kuhn and D. v. Oheimb. Interacting state machines for mobility. In K. Araki, S. Gnesi, and D. Mandrioli, editors, Proc. 12th Intl. FME Symposium (FM2003), volume 2805 of Lect. Notes in Comp. Sci., pages 698-718, Pisa, Italy, Sept. 2003. Springer-Verlag.

9. L. Lamport. The Temporal Logic of Actions. ACM Trans. Prog. Lang. Syst., 16(3):872-923, May 1994.

10. D. Latella, M. Massink, H. Baumeister, and M. Wirsing. Mobile UML statecharts with localities. Technical report 37, CNR ISTI, Pisa, Italy, 2003.

11. S. Merz, J. Zappe, and M. Wirsing. A spatio-temporal logic for the specification and refinement of mobile systems. In M. Pezzè, editor, Fundamental Approaches to Software Engineering (FASE 2003), volume 2621 of Lect. Notes in Comp. Sci., pages 87-101, Warsaw, Poland, April 2003. Springer-Verlag.

12. R. D. Nicola, G. Ferrari, and R. Pugliese. Klaim: a kernel language for agents interaction and mobility. IEEE Trans. Software Eng., 24(5):315-330, 1998.

13. R. D. Nicola and M. Loreti. A modal logic for Klaim. In T. Rus, editor, Algebraic Methodology and Software Technology (AMAST 2000), volume 1816 of Lect. Notes in Comp. Sci., pages 339-354, Iowa, 2000. Springer-Verlag.

14. Object Management Group. Unified Modeling Language Specification, Version 1.5. Specification, OMG, 2003. http://cgi.omg.org/cgi-bin/doc? formal/03-03-01.

15. B. Paech and B. Rumpe. A new concept of refinement used for behaviour modelling with automata. In Formal Methods Europe (FME'94), volume 873 of Lect. Notes in Comp. Sci., pages 154-174, Barcelona, Spain, 1994. Springer-Verlag.

16. P. Scholz. A refinement calculus for Statecharts. In Fundamental Approaches to Software Engineering (FASE'98), volume 1382 of Lect. Notes in Comp. Sci., pages 285-301, Lisbon, Portugal, 1998. Springer-Verlag.

17. M. Schrefl and M. Stumptner. Behavior-consistent specialization of object life cycles. ACM Trans. Software Eng. Meth., 11(1):92-148, 2002.

18. M. von der Beeck. Formalization of UML-statecharts. In M. Gogolla and C. Kobryn, editors, Proc. 4th Int. Conf. UML (UML 2001), volume 2185 of Lect. Notes in Comp. Sci., pages 406-421. Springer, 2001. 